with shingles, genital herpes, and those at risk for CMV infection. There are eight multinational Phase 2/3 studies under way involving sites in the United States, Canada, Europe, and Australia.

\section{WHO Aims to Eliminate Leprosy}

The World Health Organization (WHO) has set a goal of eliminating leprosy by the end of the century. An investment of $\$ 420$ million would be needed to eliminate the disease by the year 2000-one third of the amount needed for multidrug therapies (MDT).

Leprosy currently affects an estimated 3.1 million people worldwide; 2.3 million of those cases are currently undergoing treatment, with 600,000 new cases annually. India accounts for $64 \%$ of all registered cases. Brazil, Indonesia, Myanmar (Burma), and Nigeria account for another $17 \%$. WHO strategy calls for an increased focus on these highly endemic areas for case detection and MDT coverage.

FROM: The Nation's Health November 1993.

\section{ELISA and Antigen 60 for Diagnosis of TB in Children}

Researchers found that enzyme-linked immunosorbent assay (ELISA) using antigen 60 (A60) improves the diagnosis of TB in children and may distinguish recent infection without disease from infection with disease.

Results obtained in 31 children with active TB and in 16 patients with tuberculous infection without disease were compared with the results of 198 control subjects with no mycobacterial disease. In control children, anti-A60 IgG increased with age and the optical density (OD) in ELISA assays rose from $0.079 \pm 0.053(\mathrm{OD} \pm \mathrm{SD})$ in children younger than five years old to $0.146 \pm 0.082 \mathrm{OD}$ in children older than five years. In control subjects younger than two years old, IgG OD values were significantly higher in BCG-vaccinated children than in nonvaccinated children. At a chosen specificity of $98 \%$, a positive serodiagnosis was observed in $68 \%$ of children with clinically active tuberculosis. In these children with active disease, smears were positive in only $26 \%$ of cases and mycobacterial cultures yielded the organism in $45 \%$ of cases. None of the infected children without disease had high IgG OD values.

IgM measurements also were evaluated. Mean values from control and diseased children overlapped, leading to a low sensitivity (19\%) in children with clinically active tuberculosis. The authors concluded that anti-A60 IgG measure is a rapid and low-cost technique that enhances the diagnosis of clinically active tuberculosis in children and may distinguish recent infection without disease from infection with disease.

FROM: Rooney MW, Hirsch LJ, Mahtru M. A nesthesiology 1993;79:60-63.

Additional news items in this issue: International Conference on the Prevention of Infection (page 11), FDA Issues Alert on $\mathrm{H}$ emodialysis W ater Q uality Systems (page 21), Lab Errors Responsible for Some False-Positive Multidrug-Resistant TB Cases during Outbreaks (page 26), TB Transmitted to $\mathrm{H}$ ealthcare Worker from Intubated $\mathrm{N}$ eonate (page 31 ). 